\title{
Plasmonic Properties of Nanostructured Diamond Like Carbon/Silver Nanocomposite Films with Nanohole Arrays
}

\author{
Šarūnas MEŠKINIS ${ }^{1}$ *, Iryna YAREMCHUK ${ }^{2}$, Viktoras GRIGALIŪNAS ${ }^{1}$, \\ Andrius VASILIAUSKAS ${ }^{1}$, Arvydas ČIEGIS ${ }^{1}$
}

${ }^{1}$ Institute of Materials Science of Kaunas University of Technology, Baršausko 59, LT-51423 Kaunas, Lithuania
${ }^{2}$ Department of Photonics, Lviv Polytechnic National University, S. Bandera Str. 12, Lviv 79013, Ukraine

cross $^{\text {ref }}$ http://dx.doi.org/10.5755/j01.ms.22.4.13193

Received 21 September 2015; accepted 01 October 2015

\begin{abstract}
Plasmonic properties of the diamond like carbon nanocomposite films with embedded silver nanoparticles with patterned nanohole arrays were analyzed in this study. The films were deposited by unbalanced reactive magnetron sputtering of silver target. Nanopatterning of the films was performed by combining electron beam nanolithography and ion beam etching techniques. Modeling of plasmonic properties was done using the classical Maxwell-Garnett theory. Modeling data and experimental results were in good accordance. Formation of the nanohole pattern in diamond like carbon films doped with silver resulted in decreased intensity of the surface plasmon resonance absorbance peak. No new absorbance or transmittance peaks were observed after the nanopattering. It was explained by extraordinary transmission effect in nanostructured DLC : Ag film films due to plasmon polariton resonance inside of the nanoholes.

Keywords: plasmon polariton resonance, diamond like carbon films, nanohole array.
\end{abstract}

\section{INTRODUCTION}

Nanocomposites consisting of the dielectric matrix with embedded group IB metal ( $\mathrm{Au}, \mathrm{Ag}, \mathrm{Cu}$ ) nanoparticles received considerable interest of the researchers $[1,2]$. Particularly presence of the $\mathrm{Au}, \mathrm{Ag}$ or $\mathrm{Cu}$ nanoparticles results in appearance of the surface plasmon resonance effect in such nanocomposite films $[1,2]$. On the other hand, plasmonic properties of the nanocomposite can be additionally controlled by using dielectric matrix of the appropriate refractive index [2]. Besides that, dielectric matrix protects metal nanoparticles from the unwanted environmental effects such as oxidation. In such a way main disadvantage of the $\mathrm{Au}$ nanoparticles - oxidation resistance [3] - can be successfully eliminated enabling exploitation advantages of other group IB metals such as stronger surface plasmon resonance effect in the case of silver [3, 4]. Another important advantage of the plasmonic nanocomposites in comparison with nanoparticles is their compatibility with semiconductor device fabrication technology. Particularly, compatibility with nanofabrication techniques such as electron beam and UV lithography as well as ion beam and reactive ion etching can be mentioned [5]. In such a way, in principle different plasmonic nanocomposite based nanostructures (metamaterials) such as nanodots, split rings, nanoholes, gratings can be fabricated. However, there are few studies on this matter. Therefore, in this article nanohole arrays were fabricated in the plasmonic diamond like carbon nanocomposites with embedded silver nanoparticles (DLC: Ag). Optical properties of the fabricated nanostructures were investigated.

\footnotetext{
${ }^{*}$ Corresponding author. Tel.: + 370-37-313432; fax.: +370-37-314423

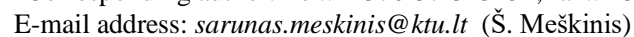

\section{EXPERIMENTAL TECHNIQUES}

Fabrication process of diamond like carbon nanocomposite films consists of a few steps - deposition, patterning and etching. Deposition of the silver doped diamond like carbon nanocomposite film was done by DC unbalanced reactive magnetron sputtering of silver target. The diameter of magnetron was 3 inches. Fused silica substrates were used. Mixture of the hydrocarbons (acetylene) and argon gas was used in the reactive magnetron sputtering. Ar gas flux was $80 \mathrm{sccm}$ and $\mathrm{C}_{2} \mathrm{H}_{2}$ gas flux was $7.8 \mathrm{sccm}$. In all experiments substrate - target gap was set at $10 \mathrm{~cm}$, magnetron target current was $0.1 \mathrm{~A}$, base pressure was $5 \times 10^{-4} \mathrm{~Pa}$ and work pressure was $(4 \pm 1) \times 10^{-1} \mathrm{~Pa}$. The thickness of deposited films was about $50 \mathrm{~nm}$. No additional bias was used, and substrates were grounded. Nanopatterning of the silver doped diamond like carbon nanocomposite film was done using Raith e-LiNE ${ }^{\text {lus }}$ e-beam nanolithography \& scanning electron microscopy system that includes a column and electron source designed to provide a Gaussian beam of $2 \mathrm{~nm}$ size (at $20 \mathrm{kV}$ with a probe current of $300 \mathrm{pA}$, current density at this smallest beam size is equal to $7500 \mathrm{~A} / \mathrm{cm}^{2}$ ). Beam voltage can be selected within the range from $20 \mathrm{~V}$ to $30 \mathrm{kV}$ in steps of $10 \mathrm{~V}$, working distance and seven beam defining aperture settings can be adjusted separately. The apertures are changed electromagnetically providing a current range from $5 \mathrm{pA}$ to approximately $20 \mathrm{nA}$. Micro- and nanostructures in Fig. 1 -Fig. 3 were fabricated using a single layer of high molecular weight $(950 \mathrm{~K})$ polymethyl methacrylate (PMMA) dissolved in Anisole at $4 \%$ solids. PMMA was spin coated at $3000 \mathrm{rpm}$ for 30 seconds and dried for 2 minutes at $200{ }^{\circ} \mathrm{C}$ on hotplate (the thickness of spincoated layer was measured by laser ellipsometer Gaertner L-115 and varied from 230 to $240 \mathrm{~nm}$ ). Electron beam 
exposure was done at $10 \mathrm{kV}$ (aperture diameter $30 \mu \mathrm{m}$, probe current $250 \mathrm{pA}$, write field size $100 \times 100 \mu \mathrm{m}$ ) with dose variations from 120 to $140 \mu \mathrm{C} / \mathrm{cm}^{2}$. Exposed PMMA was developed in methyl isobutyl ketone (MIBK) and isopropanol (IPA) solution $(1: 3)$ for 30 seconds with agitation (development was stopped in isopropyl alcohol for 15 seconds and the sample dried at room temperature with dry nitrogen flow). After the fabrication of the nanoholes array in PMMA by e-beam nanolithography, Ar ion beam etching of DLC : Ag film followed. Argon ion beam etching was performed by using ion beam etching unit "Usi-ionic" (LPA Industry) equipped with a multi-cell closed drift Hall-current ion source. The following technological process parameters were used: ion beam energy $300 \mathrm{eV}$, ion current density $0.25 \mathrm{~mA} / \mathrm{cm}^{2}$, work pressure $1 \times 10^{-1} \mathrm{~Pa}$, substrate temperature $20^{\circ} \mathrm{C}$. After etching, PMMA mask was removed in Remover PG (Micro Resist Technology GmbH), as shown in Fig. 5.

Optical properties of the nanopatterned DLC : Ag film were evaluated by AvaSpec-2048 spectrometer in the ultraviolet and visible (UV-VIS) region. Spectrometer is based on the AvaBench-75 symmetrical Czerny-Turner design and is equipped with 2048 pixel CCD detector array (resolution $-1.4 \mathrm{~nm}$ ).

Volume filling factor was calculated as a ratio between the volume of the nanoholes and overall volume of the film.

\section{EXPERIMENTAL RESULTS}

Electron beam lithography presents the smallest writing tool known and is the preferred technology when the highest resolution is required to fabricate nanometerscale structures. Unfortunately, due to extremely small beam spot, electron beam lithography technique is up to 10 million times slower than optical lithography and this is a serious limitation, especially when writing many small patterns over a large area. Efficient nanoscale patterning requires precise focusing and alignment procedures and system parameters shall be customized for specific applications, e.g. exposing different parts of one design and changing column and other parameters in between [6]. Resolution of electron beam lithography is determined not only by the focused beam size, but also very much depends on electron beam resist thickness, electron scattering and proximity effects (it is almost impossible to fabricate structures of size less than electron beam resist thickness). Having resist thickness of $230-240 \mathrm{~nm}$, we have chosen four patterning dimensions of plasmonic nanoholes array, varying patterned area from $100 \times 100$ to $400 \times 400 \mathrm{~nm}$, and varying spacing between patterned holes from 300 to $600 \mathrm{~nm}$. Dimensions of the plasmonic nanoholes array are listed in Table 1.

Table 1. Dimensions of the plasmonic nanoholes array

\begin{tabular}{|c|c|c|c|c|}
\hline Title & $\begin{array}{c}\text { Patterned hole } \\
\text { dimension, nm }\end{array}$ & $\begin{array}{c}\text { Spacing between } \\
\text { patterned holes, } \\
\text { nm }\end{array}$ & $\begin{array}{c}\text { Period, } \\
\text { nm }\end{array}$ & $\begin{array}{c}\text { Volume } \\
\text { filling } \\
\text { factor } f\end{array}$ \\
\hline E100_T300 & $100 \times 100$ & 300 & 400 & 0.07 \\
\hline E200_T400 & $200 \times 200$ & 400 & 600 & 0.27 \\
\hline E300_T300 & $300 \times 300$ & 300 & 600 & 0.44 \\
\hline E400_T600 & $400 \times 400$ & 600 & 1000 & 0.28 \\
\hline
\end{tabular}

Micrographs of plasmonic nanoholes array fabricated in the PMMA coated onto the silver doped diamond like carbon nanocomposite films are shown in Fig. 1 and Fig. 2. Fig. 1 illustrates array with patterning dimensions of 200 x $200 \mathrm{~nm}$. Nanohole diameter expands here to $350 \mathrm{~nm}$, respectively spacing between patterned holes shrinks to $250 \mathrm{~nm}$. Fig. 2 illustrates array with patterning dimensions of $300 \times 300 \mathrm{~nm}$. Actual nanohole diameter here is equal to $450 \mathrm{~nm}$, and actual spacing between patterned holes is $150 \mathrm{~nm}$. There is good accordance between dimensions of the nanoholes fabricated in PMMA layer and dimension of the nanoholes fabricated in DLC:Ag film (Fig. 2, Fig. 3).

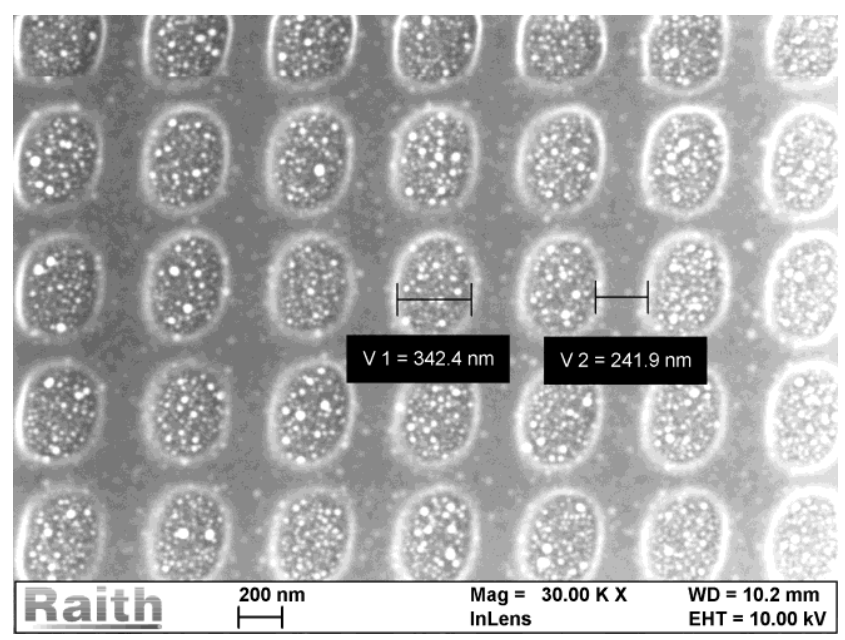

Fig. 1. SEM micrograph illustrates patterned hole array (patterning dimensions $200 \times 200 \mathrm{~nm}$, spacing $400 \mathrm{~nm}$ ) fabricated in PMMA layer, spin coated on the silver doped diamond like carbon nanocomposite film

Absorbance spectra of the patterned silver doped diamond like carbon nanocomposite films in the ultraviolet and visible range are presented in Fig. 4. Original surface plasmon resonance peak is seen at $455 \mathrm{~nm}$ in all patterning cases, except the smallest holes' case of $100 \mathrm{x} 100 \mathrm{~nm}$. This case can be attributed to the ion beam over-etching and PMMA mask destroying.

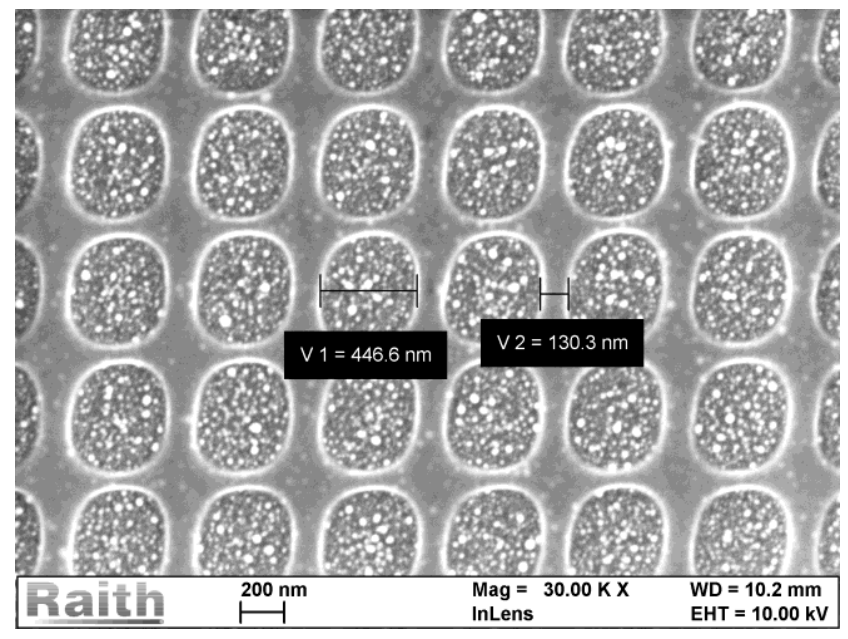

Fig. 2. SEM micrograph illustrates patterned hole array (patterning dimensions $300 \times 300 \mathrm{~nm}$, spacing $300 \mathrm{~nm}$ ) fabricated in PMMA layer, spin-coated on the silver doped diamond like carbon nanocomposite film 
The smallest and the most broadened peak can be seen in the case of patterning $200 \times 200 \mathrm{~nm}$ with spacing between holes of $400 \mathrm{~nm}$ (after ion beam etching hole diameter is expanded about to $350 \mathrm{~nm}$, and spacing shrinked to $250 \mathrm{~nm}$, see Fig. 1).

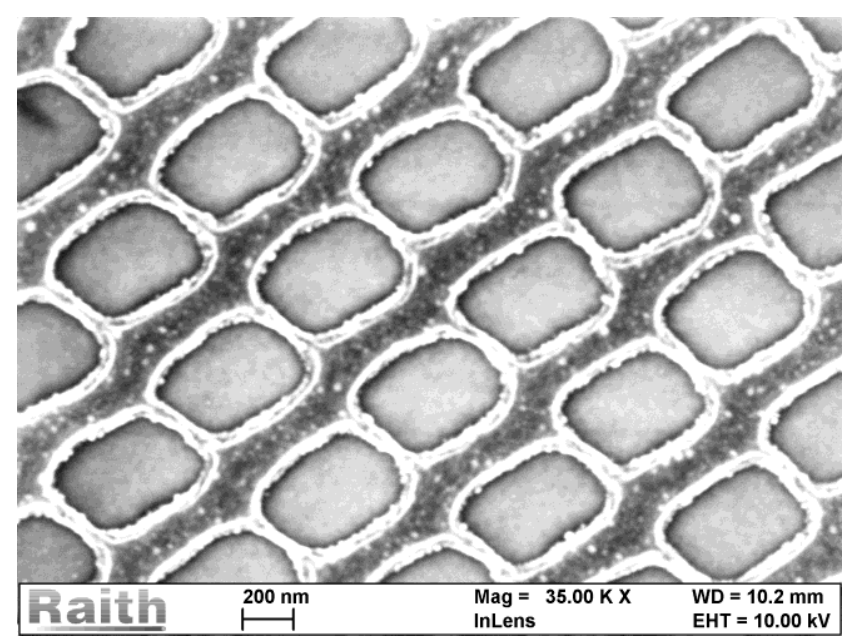

Fig. 3. SEM micrograph illustrates patterned silver doped diamond like carbon nanocomposite film after removal of PMMA

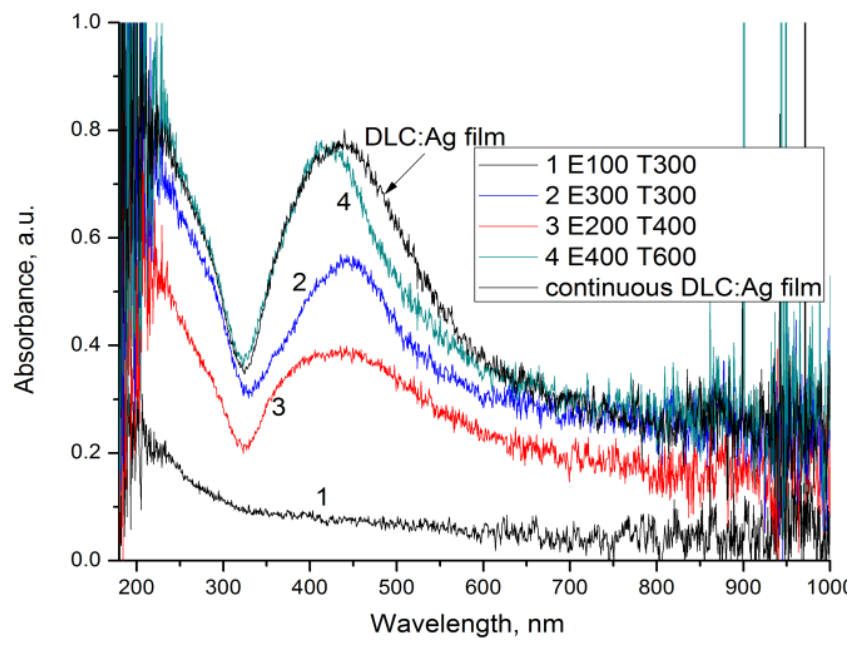

Fig. 4. Absorption spectra of the patterned silver doped diamond like carbon nanocomposite films

The peak intensity is about twice less than in the case of the unpatterned silver doped diamond like carbon film. Increasing (see Fig. 2) the hole diameter and shrinking spacing between holes (the period is kept the same $600 \mathrm{~nm}$ ) plasmon resonance peak intensity apparently increases. In the case of hole diameter $400 \times 400 \mathrm{~nm}$ with spacing between the holes of $600 \mathrm{~nm}$ plasmon resonance peak intensity is almost the same, as in the case of unpatterned silver doped diamond like carbon film, but the peak location is slightly shifted to shorter wavelengths (blue-shift). It is obvious, that variations of silver doped diamond like carbon film patterning dimensions has strong impact on plasmon resonance peak intensity and also can effect peak wavelength. However, no additional absorbance peaks appear as a result of the nanopatterning.
Ratio between absorbance of the nanostructures DLC : Ag with nanohole pattern and absorbance of the continuous DLC:Ag film for more thorough analysis of the absorbance spectra is presented in Fig. 5. It can be seen that in the case of the nanostructured DLC:Ag film containing $400 \mathrm{~nm}$ diameter nanoholes absorbance decreases only in the region of the surface plasmon resonance peak. In other cases, absorbance decrease in all analyzed range of the spectra.

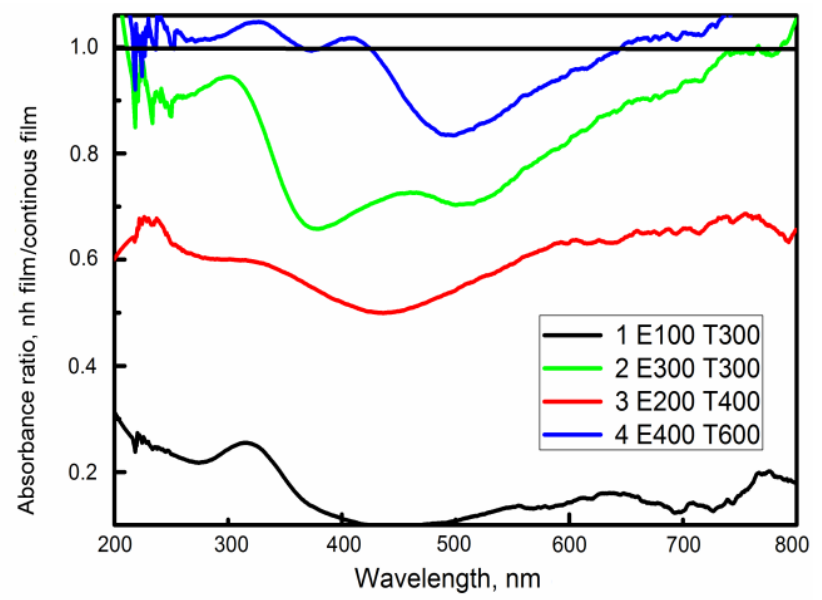

Fig. 5. Ratio of absorbance of the patterned and continuous silver doped diamond like carbon nanocomposite film

However, in all cases the highest decrease of the absorbance takes place in the region of the surface plasmon resonance peak. Nanohole nanopattering of DLC:Ag films can result in decreased absorbance due to the two reasons. If light transmission is increased due to the geometric effect, absorbance should decrease in all spectral range [7]. Such decrease is seen for 3 samples. It seems that geometric effect is absent only in the case of the nanopatterned film containing largest nanoholes. The second reason can be extraordinary transmission effect already reported for nanostructured plasmonic metal films with nanohole arrays [8,9]. Extraordinary transmission effect occurs due to plasmon polariton resonance inside of nanoholes for TM polarization. In our case this resonance occurs in addition to the local plasmon resonance in $\mathrm{Ag}$ nanoparticles. It seems that due to the high concentration of silver particles and interactions between them plasmon polariton wave occurs in the holes of the nanocomposite. In all cases extraordinary transmission effect occurs in all samples.

\section{THEORETICAL CONSIDERATIONS}

Pendry et al [10] have shown that for a surface wave that propagates along a perforated metal film one is able to introduce an effective permittivity. The effective dielectric permittivity of 2D arrays of nanoapertures in opaque metal films has the form identical to that of plasmonic materials. Thus, we can consider the silver doped nanocomposite diamond like carbon (DLC-Ag) films with the holes' array as plasmonic crystal with its period much smaller than the operating wavelength. Such structure behaves as an 
effective medium with its optical parameters dependent on its design.

The classical effective Maxwell-Garnett theory [11] was used for calculation of the optical characteristics of DLC-Ag film with nanoholes array:

$$
\varepsilon_{e f f_{-} 2 D}=\varepsilon_{a i r} \frac{\varepsilon_{D L C-A g}(1+2 f)+2 \varepsilon_{a i r}(1-f)}{\varepsilon_{D L C-A g}(1-f)+\varepsilon_{a i r}(2+f)} .
$$

Volume filling factor $(f)$ of the air inclusions was calculated by analogy to diffraction 1D gratings. The optical constants of the nanocomposite film ( $\left.\varepsilon_{\text {DLC-Ag }}\right)$ were modeled using the renormalized Maxwell-Garnett theory [12] by taking into account the dipole-dipole interactions between particles. The optical properties of silver are obtained from the experimental data by Johnson and Christy [13]. The refractive index $(n)$ and extinction coefficient $(k)$ dispersion curves of DLC film are used from [14]. The average particle size of silver nanoparticles is $10 \mathrm{~nm}$ according to [15]. Application of this effective theory provides good fit of the experimental refractive index and absorption spectra of the DLC-Ag films with the silver nanoparticle concentration up to $22 \%$ [15].

Fig. 8 shows modelled and experimental absorption spectra of the various nanoholes arrays patterned silver doped diamond like carbon nanocomposite films. Comparison of the modelled and experimental curves shows good agreement between the theoretical and experimental data, consequently, this model can be used to predict plasmonic behaviour of patterned medium.

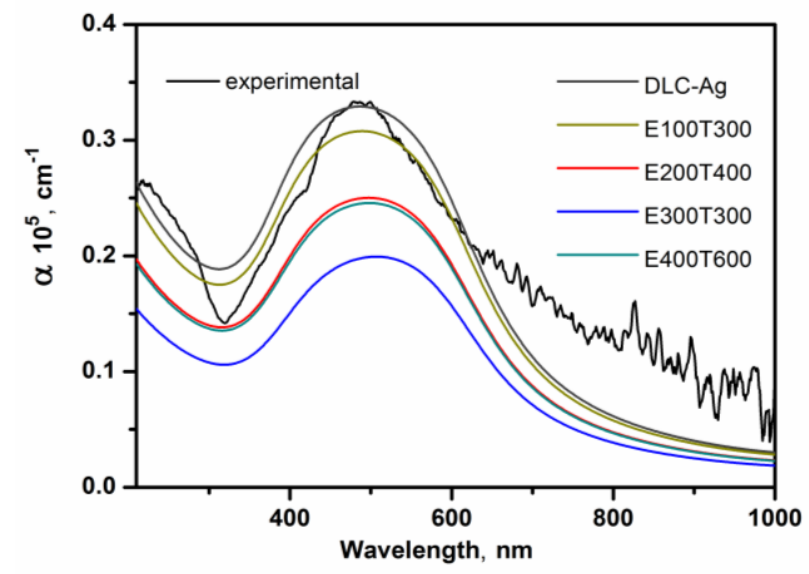

Fig. 8. Modelled and experimental absorption spectra of the patterned silver doped diamond like carbon nanocomposite films

It can be seen that overall tendencies are the same both for modelled and experimentally measured aborbance spectra of nanopatterned DLC:Ag films. In all cases extraordinary transmittance effect related decrease of the intensity of plasmonic peak can be seen (Fig. 6-Fig. 8). Similarly, to the experimental data modeling shows presence of the geometric effect, too.

\section{CONCLUSIONS}

Silver doped diamond like carbon nanocomposite films were patterned by micro- and nanosize hole arrays using e-beam nanolithography and argon ion beam etching system. Formation of the nanohole pattern in DLC : Ag films resulted in decreased intensity of the plasmonic peak. In some cases, small shift of the peak position as well as some changes of the peak width was observed. No new absorbance or transmittance peaks were detected. It was explained by appearance of the extraordinary transmission effect in nanostructured films due to plasmon polariton resonance inside of the nanoholes fabricated in the plasmonic DLC:Ag film. Competition between to plasmon polariton resonance in nanoholes and local plasmon resonance in $\mathrm{Ag}$ nanoparticles results in decreased intensity of the surface plasmon resonance related absorbance peak.

For part of the samples overall decrease of the absorbance in all investigated wavelength range was observed. It was explained by geometric effect related with nanostructuring of DLC : Ag films.

Simulation performed using classical effective Maxwell-Garnett theory and modelling test structures were in good accordance with the experimental results and supported presence of the extraordinary transmittance effect in all samples investigated.

\section{Acknowledgement}

This research was funded by the European Social Fund under the Global Grant measure (project No. VP1-3.1ŠMM-07-K-03-057). Support of the Research Council of Lithuania in the frames of the Lithuania-Ukraine research collaboration program should be acknowledged (grant TAP LU 04/2014).

\section{REFERENCES}

1. Etrich, Ch., Fahr, S., Hedayati Mehdi, K., Faupel, F., Elbahri, M., Rockstuhl, C. Effective Optical Properties of Plasmonic Nanocomposites Materials 7 2014: pp. $727-741$

2. Hedayati, M.K., Faupel, F., Elbahri, M. Review of Plasmonic Nanocomposite Metamaterial Absorber Materials 7 (2) 2014: pp. 1221-1248.

3. Pena-Rodríguez, O., Pal, U. Enhanced Plasmonic Behavior of Bimetallic ( $\mathrm{Ag}-\mathrm{Au})$ Multilayered Spheres Nanoscale Research Letters 6 2011: pp. 279-284. http://dx.doi.org/10.1186/1556-276X-6-279

4. Kyeong-Seok L., El-Sayed, M.A. Gold and Silver Nanoparticles in Sensing and Imaging: Sensitivity of Plasmon Response to Size, Shape, and Metal Composition Journal of Physical Chemistry B 110 (39) 2006: pp. $19220-19225$.

5. Tamulevičius, T., Tamulevičienè, A., Virganavičius, D., Vasiliauskas, A., Kopustinskas, V., Meškinis, Š, Tamulevičius, S. Structuring of DLC : Ag Nanocomposite Thin Films Employing Plasma Chemical Etching And Ion Sputtering Nuclear Instruments and Methods in Physics Research (B) 341 2014: pp. 1-6.

6. Højlund-Nielsen, E., Greibe, T., Asger Mortensen, N., Kristensen, A. Single-spot E-beam Lithography for Defining Large Arrays of Nano-holes Microelectronic Engineering 121 2014: pp. 104-107.

7. Chih, W., Hsuan, I.W., Chih-Wei, L., Jihperng, L. Anisotropic Optical Transmission of Femtosecond Laser Induced Periodic Surface Nanostructures on 
Indium-Tin-Oxide Films Applied Physics Letters 101 (10) 2012: pp. 1-5.

8. Reilly, T.H., Tenent, R.C., Barnes, T.M., Rowlen, K.L., van de Lagemaat, J. Controlling the Optical Properties of Plasmonic Disordered Nanohole Silver Films ACS Nano 4 (2) 2010: pp. 615-624.

9. Gordon, R. Bethe's Theory for Aperture Arrays Physical Review A 76 2007: pp. 1-5.

10. Pendry, J.B., Martín-Moreno, L., Garcia-Vidal, F.J. Mimicking Surface Plasmons with Structured Surfaces Science 3055685 2004: pp. 847-848.

11. Maxwell Garnett, J.C. Colours in Metal Glasses, in Metallic Films, And in Metallic Solutions II Philosophical Transactions Royal Society London Seria A 1904: pp. 237-288.

12. Vasilevskiy, M. Effective Dielectric Response of Composites Containing Uniaxial Inclusions Physica Status Solidi (b) 219 2000: pp. 197-204. http://dx.doi.org/10.1002/15213951(200005)219:1<197::AID-PSSB197>3.0.CO;2-5

13. Johnson, P, Christy, R. Optical Constants of the Noble Metals Physical Review B 6 1972: pp. 4370-4380.

14. Yaremchuk, I., Tamulevičienè, A., Tamulevičius, T., Šlapikas, K., Balevičius, Z., Tamulevičius, S. Modeling of the Plasmonic Properties of DLC-Ag Nanocomposite Films Physica Status Solidi (a) 211 2014: pp. 329-335.

15. Yaremchuk, I., Meškinis, Š., Fitio, V., Bobitski, Y., Šlapikas, K., Čiegis, A., $\quad$ Balevičius, Z., $\quad$ Selskis, A., Tamulevičius, S. Spectroellipsometric Characterization and Modeling of Plasmonic Diamond-Like Carbon Nanocomposite Films with Embedded Ag Nanoparticles Nanoscale Research Letters 2015:10:157, published online 2015 Apr 1.

http://dx.doi.org/10.1186/s11671-015-0854-y 